Research Article

\title{
Morphological Component Analysis-Based Perceptual Medical Image Fusion Using Convolutional Sparsity-Motivated PCNN
}

\author{
Chuangeng Tian, ${ }^{1}$ Lu Tang $\mathbb{D}^{2}{ }^{2}$ Xiao Li, ${ }^{1}$ Kaili Liu, ${ }^{1}$ and Jian Wang ${ }^{1}$ \\ ${ }^{1}$ School of Information and Electrical Engineering, Xuzhou University of Technology, Xuzhou, China \\ ${ }^{2}$ School of Medical Imaging, Xuzhou Medical University, Xuzhou, Jiangsu, China \\ Correspondence should be addressed to Lu Tang; xztanglu@xzhmu.edu.cn
}

Received 28 December 2020; Revised 24 February 2021; Accepted 18 March 2021; Published 29 March 2021

Academic Editor: Qinhu Zhang

Copyright (C) 2021 Chuangeng Tian et al. This is an open access article distributed under the Creative Commons Attribution License, which permits unrestricted use, distribution, and reproduction in any medium, provided the original work is properly cited.

\begin{abstract}
This paper proposes a perceptual medical image fusion framework based on morphological component analysis combining convolutional sparsity and pulse-coupled neural network, which is called MCA-CS-PCNN for short. Source images are first decomposed into cartoon components and texture components by morphological component analysis, and a convolutional sparse representation of cartoon layers and texture layers is produced by prelearned dictionaries. Then, convolutional sparsity is used as a stimulus to motivate the PCNN for dealing with cartoon layers and texture layers. Finally, the medical fused image is computed via combining fused cartoon layers and texture layers. Experimental results verify that the MCA-CS-PCNN model is superior to the state-of-the-art fusion strategy.
\end{abstract}

\section{Introduction}

In clinical applications, medical images include anatomical images and functional images. Anatomical images provide information of dense structures [1], for instance, X-ray computed tomography (CT) and magnetic resonance imaging (MRI). Functional images reflect information of blood flow and blood activity [2], for instance, positron emission CT (PET) and single-photon emission CT (SPECT). Medical images with single modality do not provide sufficient information in diagnosing diseases; medical image fusion (MIF) technology provides an effective method via merging medical images with different modalities into a comprehensive MIF image to aid radiologists for better diagnosis [3-5].

Many MIF algorithms have been addressed in the last dozen years. These methods include the multiscale decomposition- (MSD-) based fusion strategy [6-10], sparse representation- (SR-) based fusion strategy [11], and pulsecoupled neural network- (PCNN-) based fusion strategy $[12,13]$. To pursue satisfactory fusion performance, attempts were made to use the PCNN based on MST [14-16]. PCNN is a cat visual cortex biologically inspired neural network, which is used in medical image fusion. Huang et al. [17] integrated non-subsampled contourlet transform (NSCT) with the PCNN for SPECT and CT image fusion. Nonsubsampled shearlet transform (NSST) was combined with the PCNN to fuse medical images [18]. However, NSCT- or NSST-based fusion strategy has high computational complexity due to proper contours, which may limit the fusion performance. Furthermore, normalized coefficient values are employed to stimulate the PCNN, which may cause detail loss and blurring effect in the fused image. Electrophysiological experiments have proved that the neuron representations of complex stimulation in the cat visual cortex are represented by sparse coding [19-21]. Morphological component analysis (MCA) has been widely studied as effective image decomposition. Combining MCA with SR can acquire the SR of cartoon and texture components of an image $[22,23]$. To resolve the disadvantage produced by patch coding, convolutional sparse representation (CSR) has been shown to be more effective than sparse representation in extracting features [24]. It is implemented on the whole 


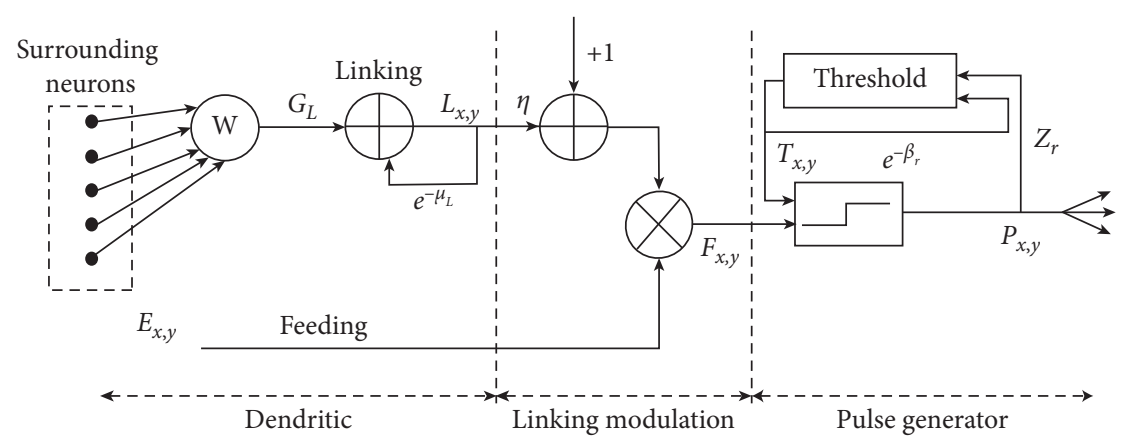

FIgURE 1: Diagrammatic diagram of the simplified PCNN.

image instead of a local image patch. Based on the above considerations, this paper presents a medical image fusion algorithm using convolutional sparsity to stimulate the PCNN based on morphological component analysis (MCA-CS-PCNN). Source images are first decomposed into cartoon components and texture components by MCA, and CSR of cartoon layers and texture layers is obtained by prelearned dictionaries. Then, convolutional sparsity is employed to stimulate the PCNN for processing cartoon layers and texture layers. The MIF image is computed via combining fused cartoon layers and texture layers. We test the performance of the proposed MCA-CS-PCNN fusion method, and the experimental results verify the advantages of our fusion strategy.

\section{Related Work}

2.1. Convolutional Sparsity Based on Morphological Component Analysis (CSMCA). Convolutional sparsity is a sparse representation model applying the convolutional form [24], which is based on an entire image rather than overlapped patch. The CSR is defined as

$$
\underset{s_{n}}{\arg \min } \frac{1}{2}\left\|I-\sum_{n=1}^{N} d_{n} \times s_{n}\right\|_{2}^{2}+\gamma \sum_{n=1}^{n}\left\|s_{n}\right\|_{1},
$$

where $I$ denotes an image, $s_{n}$ and $d_{n}$ denote the global sparse coefficient maps and dictionary filter, respectively, $*$ represents the convolution operator, and $\gamma$ is the regularization parameter.

Morphological component analysis of an image is regarded as a linear combination of different components, which is defined as [23]

$$
I=I_{c}+I_{t}
$$

where $I_{c}$ and $I_{t}$ denote cartoon components and texture components, respectively. According to CSR theory, the model of convolutional sparsity based on morphological component analysis (CSMCA) is expressed as

$$
\begin{gathered}
\underset{c_{c, n}, c_{t, n}}{\arg \min } \frac{1}{2}\left\|I-\sum_{n=1}^{n_{c}} b_{c, v} * s_{c, n}-\sum_{n=1}^{n_{t}} b_{t, n} * s_{t, n}\right\|_{2}^{2} \\
+\gamma_{c} \sum_{n=1}^{n_{c}}\left\|s_{c, n}\right\|_{1}+\gamma_{c} \sum_{n=1}^{n_{t}}\left\|s_{t, n}\right\|_{1},
\end{gathered}
$$

where $\left\{b_{c, n}\right\}_{n=1}^{n_{c}}$ and $\left\{s_{c, n}\right\}_{n=1}^{n_{c}}$ denote the dictionary and convolution sparse coefficient corresponding to $I_{c}$, respectively. $\left\{b_{t, n}\right\}_{n=1}^{n_{t}}$ and $\left\{s_{t, n}\right\}_{n=1}^{n_{c}}$ represent the dictionary and convolution sparse coefficient corresponding to $I_{t}$, respectively. The image is computed and denoted by

$$
I=\sum_{n=1}^{n_{c}} b_{c, n} * s_{c, n}+\sum_{n=1}^{n_{t}} b_{t, n} * s_{t, n} .
$$

2.2. Pulse-Coupled Neural Network. The diagrammatic diagram of the simplified PCNN is shown in Figure 1. There are three modules in the simplified PCNN model [12], which include the dendritic, linking modulation, and pulse generator, where feeding and linking input are built into the dendritic, denoted by $E_{x, y}=(m)$ and $L_{x, y}=(m)$. $F_{x, y}=(m)$ and $P_{x, y}=(m)$ denote the linking modulation and the pulse generator, respectively. The simplified PCNN model is denoted by

$$
\begin{aligned}
& E_{x, y}(m)=S_{x, y} \\
& L_{x, y}(m)=e^{-\mu_{L}} L_{x, y}(m-1)+G_{L} \sum_{u, v} W_{x, y, u, v} P_{x, y}(m-1)
\end{aligned}
$$$$
F_{x, y}(m)=E_{x, y}(m)\left[1+\eta L_{x, y}(m)\right]
$$

$T_{x, y}(m)=e^{-\beta_{T}} T_{x, y}(m-1)+Z_{T} P_{x, y}(m-1)$,$$
P_{x, y}(m)= \begin{cases}1, & F_{x, y}(m)>T_{x, y}(m), \\ 0, & T_{x, y}(m)>T_{x, y}(m),\end{cases}
$$

where $x, y$ denote pixel locations, $u, v$ represent the dislocation in the symmetric neighborhood around a pixel, $W$ and $S_{i, j}(n)$ denote the synaptic weight matrices and the external stimulus, respectively, $G_{L}$ and $\mu_{L}$ are normalizing constants, and $\eta$ varies the weight of the linking field, which denotes the linking parameter. The threshold magnitude coefficient and attenuation coefficient are represented by $Z_{T}$ and $\beta_{T}$, respectively. 


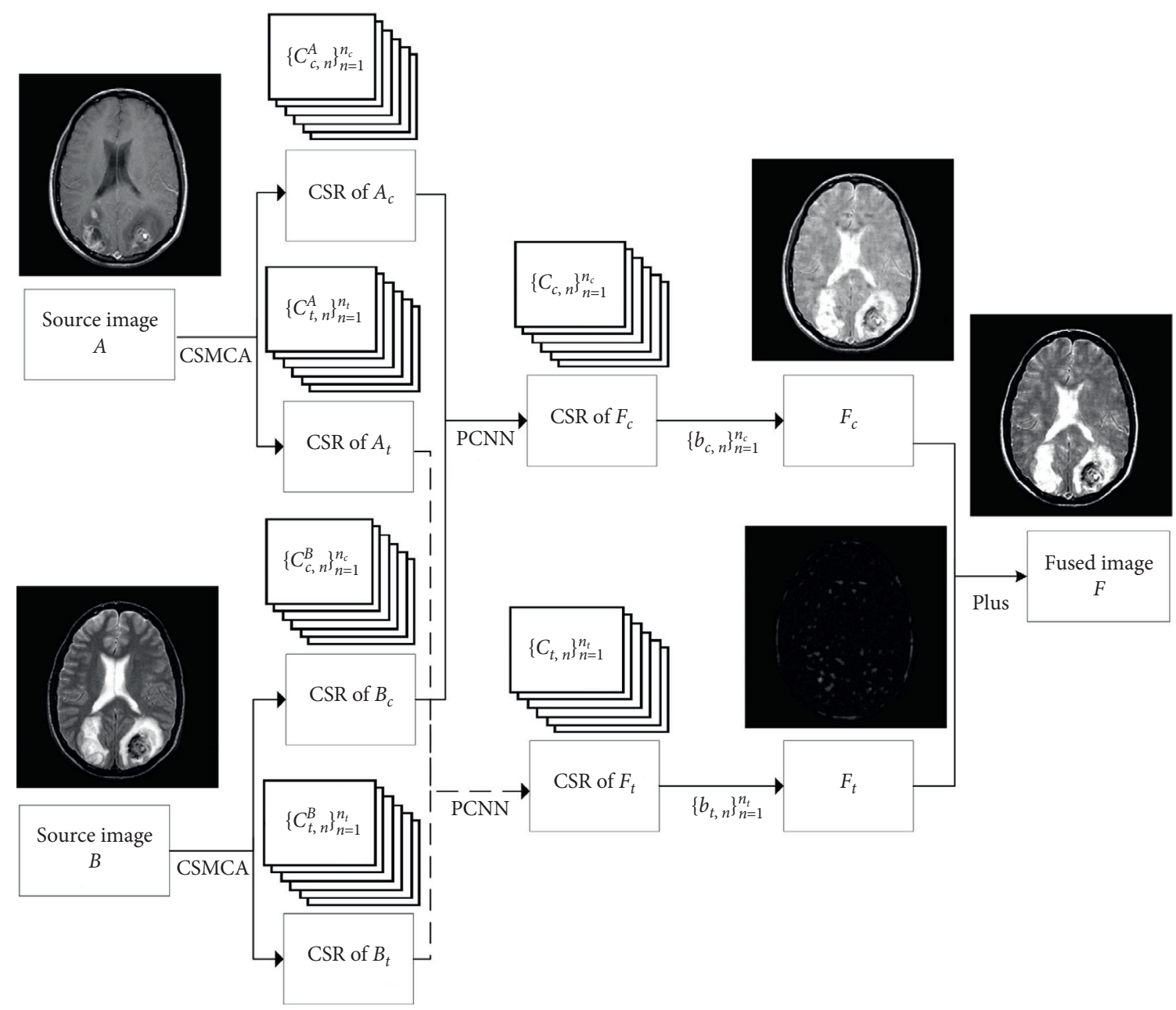

FIgURE 2: Flowchart of the anatomical and anatomical image fusion method.

\section{Proposed MIF Fusion Framework}

3.1. MCA-CS-PCNN. The flowchart of the MCA-CS-PCNN framework is shown in Figure 2. Images $A$ and $B$ denote different source images, which are decomposed into cartoon components $\left\{A^{C}, B^{C}\right\}$ and the texture components $\left\{A^{T}, B^{T}\right\}$ by applying $\mathrm{MCA}$, respectively. According to equations (1)-(4), the CSR of cartoon components and texture components is computed as

$$
\begin{aligned}
& {\left[\left\{c_{c, n}^{A}\right\}_{n=1}^{n_{c}},\left\{c_{t, n}^{A}\right\}_{n=1}^{n_{t}}\right]=\left[\left\{A_{\mathrm{CSMCA}}^{C}, A_{\mathrm{CSMCA}}^{T}\right\}\right]=\operatorname{CSMCA}(A),} \\
& {\left[\left\{c_{c, n}^{B}\right\}_{n=1}^{n_{c}},\left\{c_{t, n}^{B}\right\}_{n=1}^{n_{t}}\right]=\left[\left\{B_{\mathrm{CSMCA}}^{C}, B_{\mathrm{CSMCA}}^{T}\right\}\right]=\operatorname{CSMCA}(B),}
\end{aligned}
$$

where CSMCA $(\cdot)$ represents CSMCA functions, described in Section 2.1, $\left\{c_{c, n}^{A}\right\}_{n=1}^{n_{c}}$ and $\left\{c_{t, n}^{A}\right\}_{n=1}^{n_{t}}$ denote the convolution sparse coefficient map of $\left\{A^{C}, A^{T}\right\}$, respectively, and $\left\{c_{c, n}^{B}\right\}_{n=1}^{n_{c}}$ and $\left\{c_{t, n}^{B}\right\}_{n=1}^{n_{t}}$ denote the convolution sparse coefficient map of $\left\{B^{C}, B^{T}\right\}$.

Next, the convolutional sparse representation is used to stimulate the PCNN because complex stimulation in the cat visual cortex is based on sparse coding. $\left\{A_{\text {CSMCA }}^{C}, B_{\text {CSMCA }}^{C}\right\}$ and $\left\{A_{\mathrm{CSMCA}}^{T}, B_{\mathrm{CSMCA}}^{T}\right\}$ are employed to stimulate the PCNN for processing $\left\{A^{C}, B^{C}\right\}$ and $\left\{A^{T}, B^{T}\right\}$, respectively.

$$
\begin{aligned}
& T_{A^{C}}=\operatorname{PCNN}\left(A_{\text {CMCA }}^{C}\right), \\
& T_{B^{C}}=\operatorname{PCNN}\left(B_{\text {CSMCA }}^{C}\right), \\
& T_{A^{T}}=\operatorname{PCNN}\left(A_{\text {CSMCA }}^{T}\right), \\
& T_{B^{T}}=\operatorname{PCNN}\left(B_{\text {CSMCA }}^{T}\right),
\end{aligned}
$$

where PCNN (.) denotes the PCNN functions; the firing time matrices $\left\{T_{A^{C}}, T_{B^{C}}\right\}$ of $\left\{A^{C}, B^{C}\right\}$ and $\left\{T_{A^{T}}, T_{B^{T}}\right\}$ of $\left\{A^{T}, B^{T}\right\}$ are obtained according to equations (5)-(9), until the iteration number $m=M_{\max }$, where $M_{\max }$ denotes the max iteration times, and then iteration stops.

Then, fused coefficients of the convolution sparse coefficient map in cartoon components are computed by

$$
c_{c, n}(x, y)= \begin{cases}\max \left(c_{c, n}^{A}(x, y), c_{c, n}^{B}(x, y)\right), & T_{A^{C}}=T_{B^{C}}, \\ c_{c, n}^{A}(x, y), & T_{A^{C}}>T_{B^{C}}, \\ c_{c, n}^{B}(x, y), & T_{A^{C}}<T_{B^{C}} .\end{cases}
$$




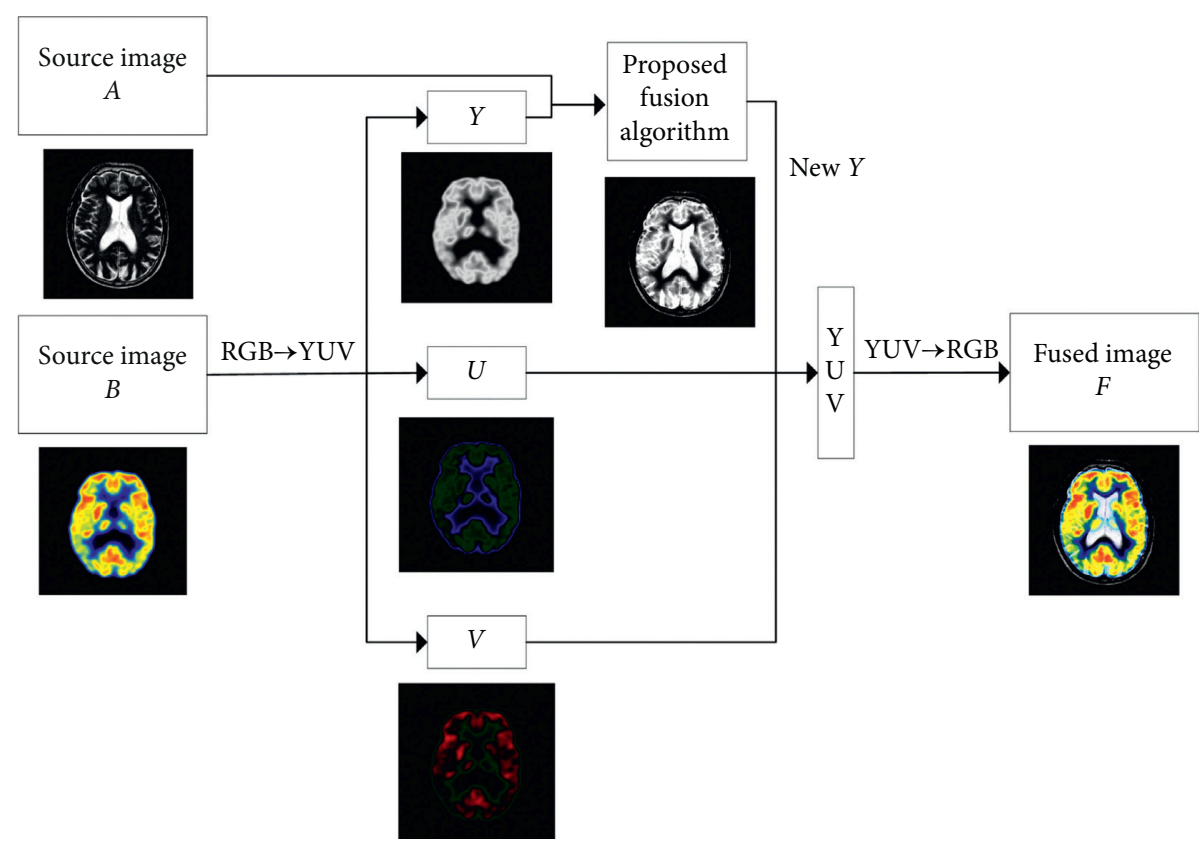

FIGURE 3: Flowchart of the anatomical and functional image fusion method.

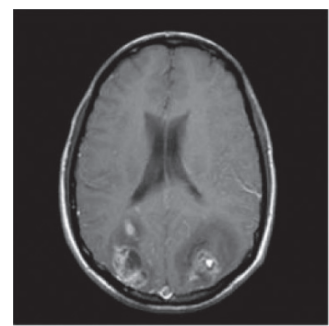

(a)

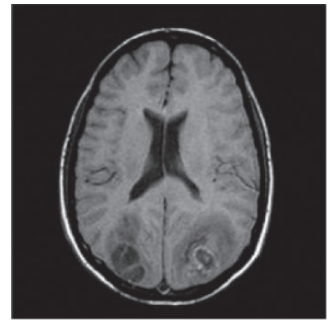

(f)

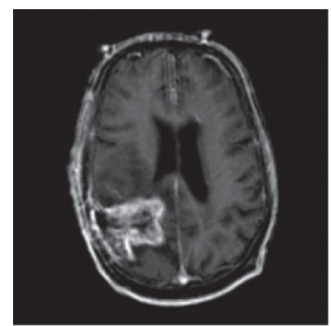

(b)

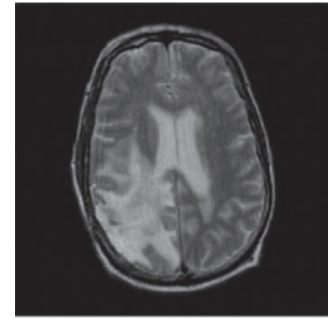

(g)

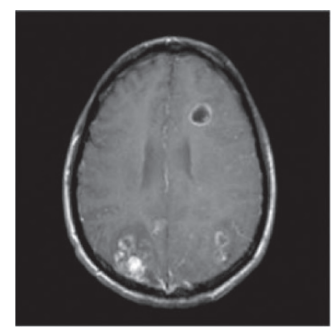

(c)

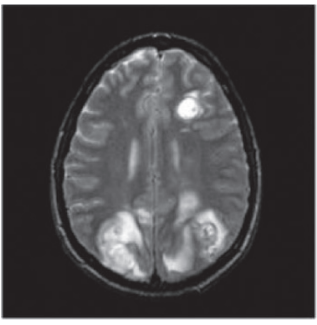

(h)

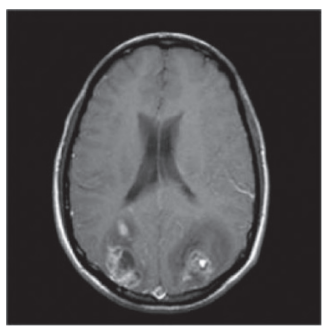

(d)

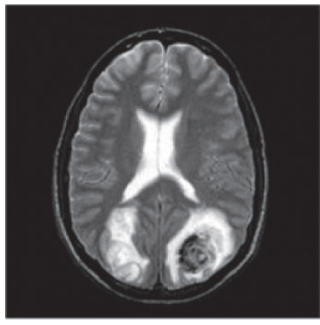

(i)

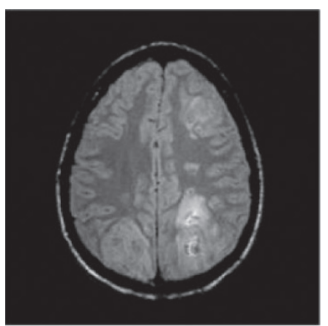

(e)

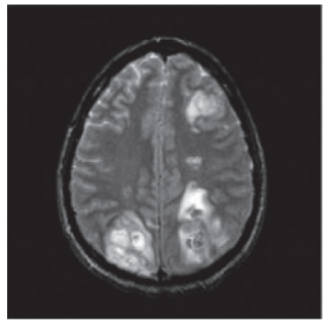

(j)

Figure 4: Five pairs of source images with anatomical and anatomical.

The fused coefficients of the convolution sparse coefficient map in texture components are computed by

$$
c_{t, n}(x, y)= \begin{cases}\max \left(c_{t, n}^{A}(x, y), c_{t, n}^{B}(x, y)\right), & T_{A^{T}}=T_{B^{T}}, \\ c_{t, n}^{A}(x, y), & T_{A^{T}}>T_{B^{T}}, \\ c_{t, n}^{B}(x, y), & T_{A^{T}}<T_{B^{T}} .\end{cases}
$$

The fused image of cartoon component $F_{c}$ and fused image of texture component $F_{t}$ are computed and denoted by

$$
\begin{aligned}
& F_{c}=\sum_{n=1}^{n_{c}} b_{c, n} * c_{c, n}, \\
& F_{t}=\sum_{n=1}^{n_{t}} b_{t, n} * c_{t, n},
\end{aligned}
$$

where $\left\{b_{c, n}\right\}_{n=1}^{n_{c}}$ and $\left\{b_{t, n}\right\}_{n=1}^{n_{t}}$ are dictionaries.

Finally, the medical fused image is acquired and denoted as

$$
F=F_{c}+F_{T}
$$




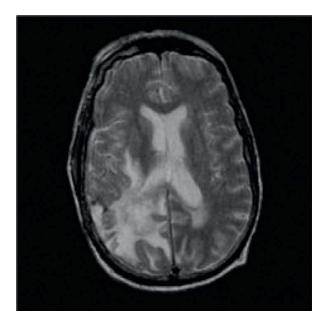

(a)

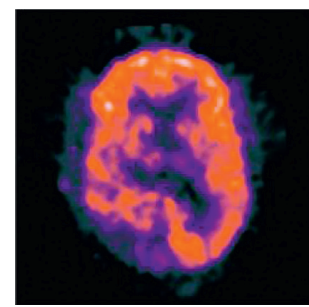

(f)

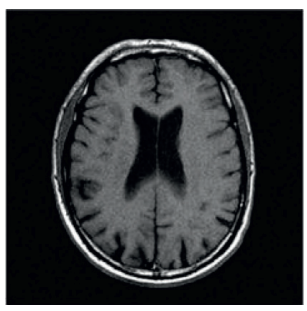

(b)

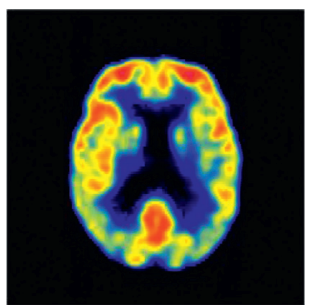

(g)

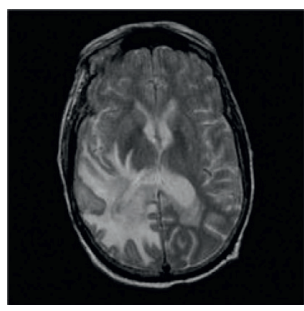

(c)

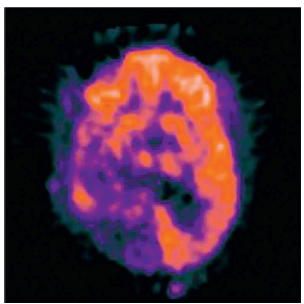

(h)

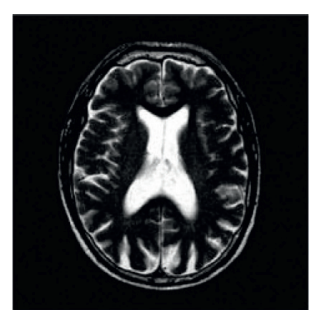

(d)

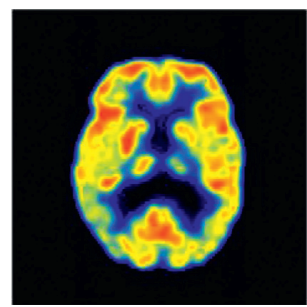

(i)

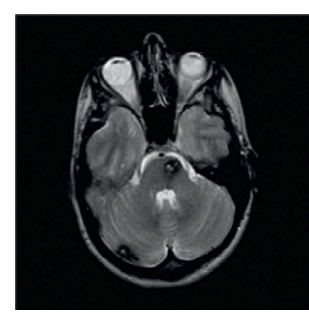

(e)

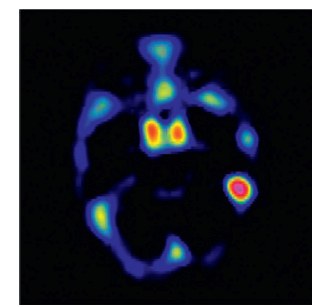

(j)

Figure 5: Five pairs of source images with anatomical and functional.
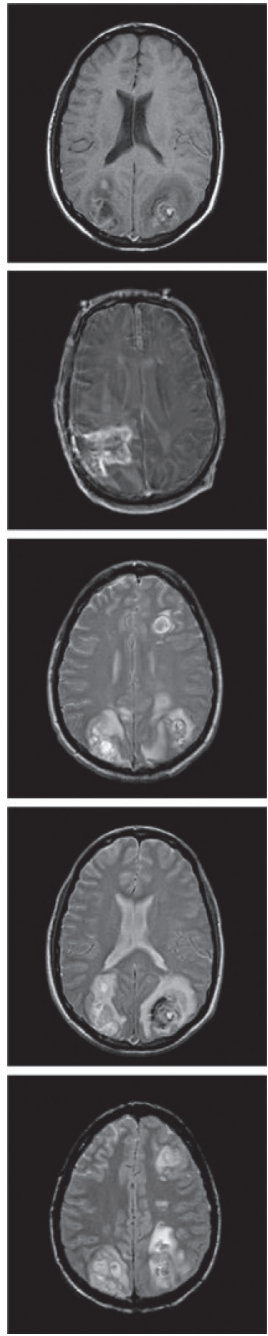

(a)
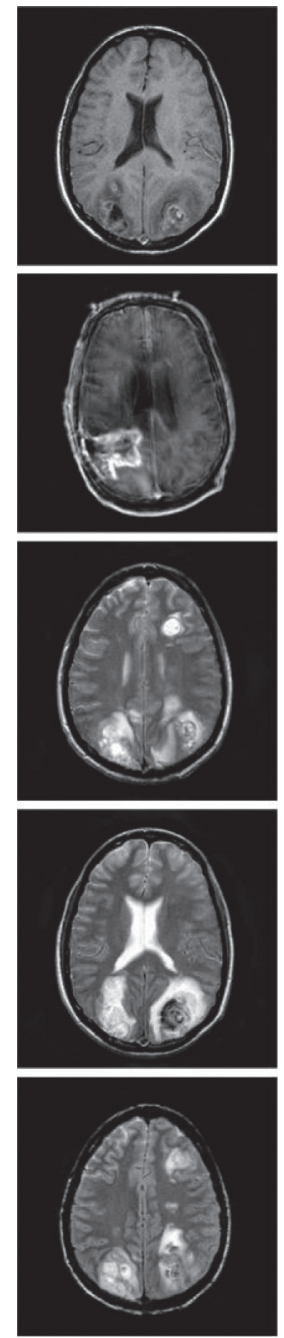

(b)
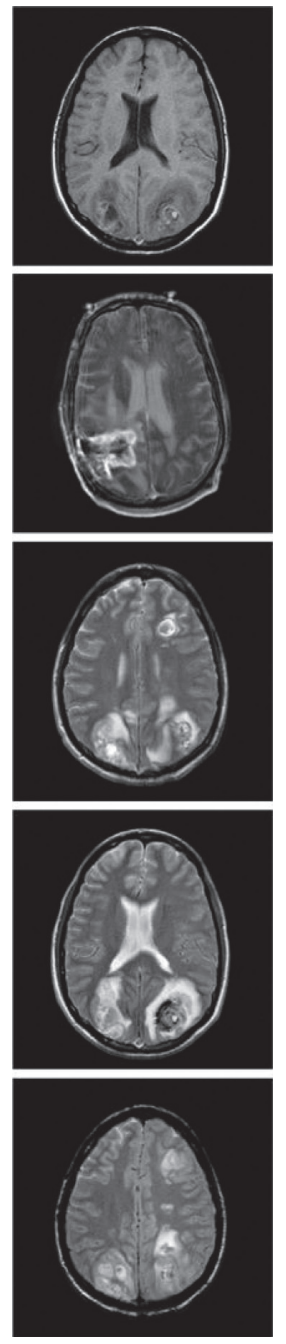

(c)
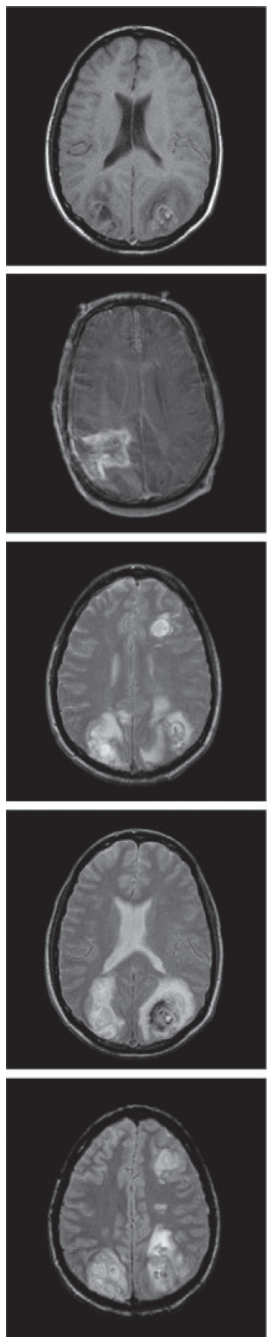

(d)
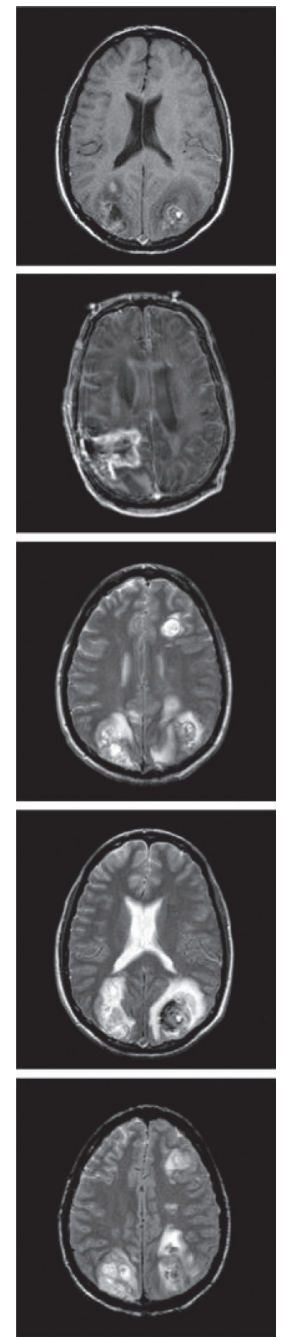

(e)
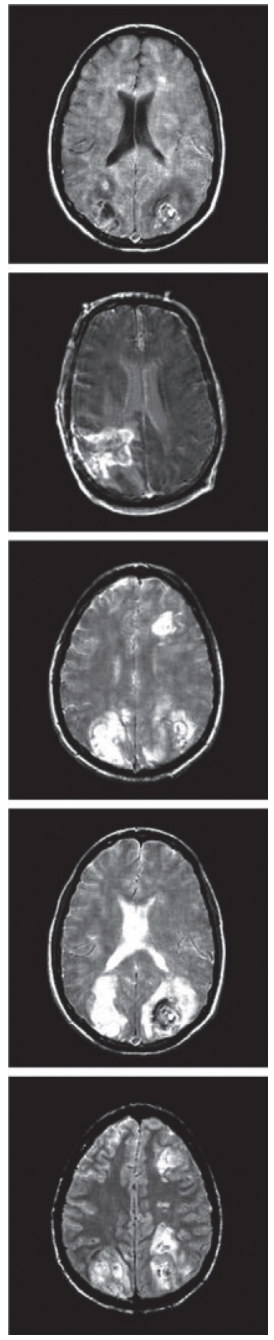

(f)

FIGURE 6: Five anatomical and anatomical image fusion results obtained by six methods. (a) CSR. (b) NSCT-MSF-PCNN. (c) GFF. (d) CSCS. (e) LP-SR. (f) Proposed. 

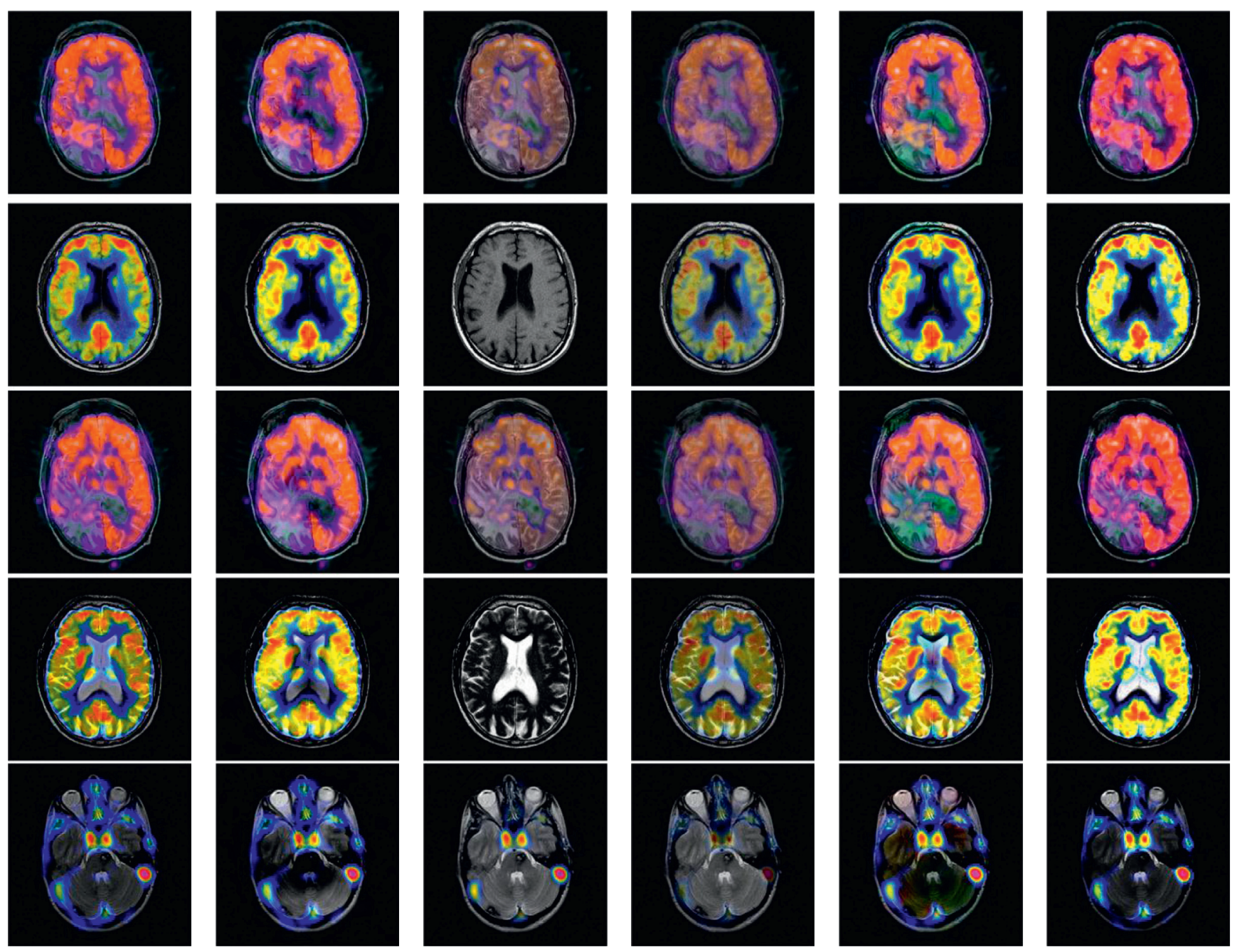

(a)

(b)

(c)

(d)

(e)

(f)

FIGURE 7: Five anatomical and functional image fusion results obtained by six methods. (a) CSR. (b) NSCT-MSF-PCNN. (c) GFF. (d) CSCS. (e) LP-SR. (f) Proposed.

3.2. Extension to Anatomical and Functional Image Fusion Based on MCA-CS-PCNN. The proposed MCA-CS-PCNN is extended to conduct anatomical and functional image fusion. Considering that functional images are pseudo-color images, the YUV color space transform has shown to be effective in processing pseudo-color images [10, 16]. Specifically speaking, a functional image with RGB is firstly transformed into the $Y$ channel, $U$ channel, and $V$ channel. Then, the new $Y$ channel is produced via the fusion of the $Y$ channel and grayscale image based on MCA-CS-PCNN, and the new YUV is acquired via merging the new $Y, U$, and $V$. Finally, YUV is converted into RGB, and the medical fused image with color is obtained. The flowchart of the anatomical and functional image fusion strategy based on MCA-CS-PCNN is shown in Figure 3.

\section{Experiments}

4.1. Experimental Settings. To test and verify the performance of the MCA-CS-PCNN fusion algorithm, ten pairs of medical images with the same size of $256 \times 256$ pixels are used to conduct the experiments, including five pairs of anatomical image and functional image fusion and five pairs of anatomical image and anatomical image fusion (Figures 4 and 5). Five representative medical image fusion algorithms are selected for experimental comparison; they are convolutional sparse representation (CSR) [24], NSCT-based modified spatial frequency and PCNN (NSCT-MSF-PCNN) [14], guided filtering (GFF) [25], cross-scale coefficient selection (CSCS) [26], and sparse representation based on the Laplacian pyramid (LP-SR) [11]. Objective quality evaluation is important for image quality [27-31]. The existing fusion quality metrics include the human perception quality metric $\left(Q_{\mathrm{HP}}\right)$ [32], feature mutual information quality metric $\left(Q_{\mathrm{FMI}}\right)$ [33], spatial frequency quality metric $\left(Q_{\mathrm{SF}}\right)$ [34], standard deviation quality metric $\left(Q_{\mathrm{SD}}\right)[11]$, nonlinear correlation information entropy metric $\left(Q_{\mathrm{NCIE}}\right)$ [35], and mutual information metric $\left(Q_{\mathrm{MI}}\right)$ [36]. In the above quality metrics, the higher the values of $Q_{\mathrm{HP}}, Q_{\mathrm{FMI}}, Q_{\mathrm{SF}}, Q_{\mathrm{SD}}, Q_{\mathrm{MI}}$, and $Q_{\mathrm{NCIE}}$, the higher the fusion performance. 
TABLE 1: Objective evaluation for anatomical and anatomical image fusion.

\begin{tabular}{|c|c|c|c|c|c|c|}
\hline Metric & CSR & NSCT-MSF-PCNN & GFF & CSCS & LP-SR & Proposed \\
\hline$Q_{\mathrm{HP}}$ & 192.5371 & 217.4689 & 204.7978 & 191.4941 & 217.2586 & 475.4320 \\
\hline$Q_{\mathrm{FMI}}$ & 0.7166 & 0.4874 & 0.7747 & 0.6542 & 0.7661 & 0.9024 \\
\hline$Q_{\mathrm{SF}}$ & 6.5501 & 6.9821 & 6.8311 & 6.7044 & 7.0135 & 7.6950 \\
\hline$Q_{\mathrm{SD}}$ & 60.8623 & 60.8988 & 61.3338 & 59.8601 & 62.0928 & 70.2132 \\
\hline$\overline{Q_{\mathrm{HP}}}$ & 877.7084 & 962.9514 & 849.0039 & 1033.4054 & 975.2739 & 1225.7900 \\
\hline$Q_{\mathrm{FMI}}$ & 0.7068 & 0.5380 & 0.7238 & 0.6372 & 0.7090 & 0.8649 \\
\hline$Q_{\mathrm{SF}}$ & 6.1220 & 6.5293 & 6.3858 & 5.6766 & 6.9076 & 6.8722 \\
\hline$Q_{\mathrm{SD}}$ & 45.4486 & 48.4487 & 49.1475 & 43.1021 & 49.6588 & 51.1843 \\
\hline$Q_{\mathrm{HP}}$ & 306.5058 & 314.8874 & 361.3098 & 424.6304 & 276.2802 & 580.2270 \\
\hline$Q_{\mathrm{FMI}}$ & 0.7600 & 0.5294 & 0.7865 & 0.6727 & 0.7750 & 0.8750 \\
\hline$Q_{\mathrm{SF}}$ & 6.0409 & 6.4068 & 6.5122 & 5.9881 & 6.6294 & 6.8274 \\
\hline$Q_{\mathrm{SD}}$ & 58.7902 & 58.1959 & 58.4755 & 56.8458 & 59.7011 & 69.6467 \\
\hline$\overline{Q_{\mathrm{HP}}}$ & 267.9495 & 317.9863 & 222.8093 & 467.2239 & 253.2109 & 481.7950 \\
\hline$Q_{\mathrm{FMI}}$ & 0.7443 & 0.4925 & 0.7747 & 0.6556 & 0.7651 & 0.8862 \\
\hline$Q_{\mathrm{SF}}$ & 6.4027 & 6.8604 & 6.8962 & 6.4012 & 7.0255 & 7.1951 \\
\hline$Q_{\mathrm{SD}}$ & 61.1738 & 67.0296 & 65.2783 & 59.9890 & 67.7791 & 78.0285 \\
\hline$\overline{Q_{\mathrm{HP}}}$ & 90.5363 & 87.7394 & 79.0995 & 195.9282 & 94.2120 & 220.7360 \\
\hline$Q_{\mathrm{FMI}}$ & 0.7467 & 0.5754 & 0.8030 & 0.7083 & 0.7741 & 0.8719 \\
\hline$Q_{\mathrm{SF}}$ & 5.8630 & 6.2027 & 5.9547 & 5.9907 & 6.3836 & 6.8953 \\
\hline$Q_{S D}$ & 55.8337 & 56.4241 & 57.1615 & 54.7563 & 56.6573 & 60.1770 \\
\hline
\end{tabular}

TABLE 2: Objective evaluation for anatomical and functional image fusion.

\begin{tabular}{|c|c|c|c|c|c|c|}
\hline Metric & CSR & NSCT-MSF-PCNN & GFF & CSCS & LP-SR & Proposed \\
\hline$Q_{\mathrm{NCIE}}$ & 0.8065 & 0.8055 & 0.8064 & 0.8067 & 0.8060 & 0.8071 \\
\hline$Q_{M I}$ & 0.6623 & 0.5171 & 0.6428 & 0.6597 & 0.6178 & 0.7081 \\
\hline$Q_{\mathrm{FMI}}$ & 0.6560 & 0.4691 & 0.6621 & 0.5600 & 0.6591 & 0.8489 \\
\hline$Q_{\mathrm{SD}}$ & 48.9095 & 53.8469 & 50.0278 & 47.3761 & 54.4485 & 55.5928 \\
\hline$Q_{\mathrm{NCIE}}$ & 0.8058 & 0.8051 & 0.8167 & 0.8058 & 0.8056 & 0.8277 \\
\hline$Q_{\mathrm{MI}}$ & 0.6760 & 0.5373 & 1.2242 & 0.6503 & 0.6640 & 0.8370 \\
\hline$Q_{\mathrm{FMI}}$ & 0.7265 & 0.4684 & 0.7894 & 0.6437 & 0.7130 & 0.8674 \\
\hline$Q_{\mathrm{SD}}$ & 59.8627 & 71.6602 & 56.7290 & 57.5273 & 73.7096 & 83.8645 \\
\hline$Q_{\mathrm{NCIE}}$ & 0.8064 & 0.8053 & 0.8063 & 0.8067 & 0.8061 & 0.8071 \\
\hline$Q_{\mathrm{MI}}$ & 0.6419 & 0.4994 & 0.6222 & 0.6417 & 0.6053 & 0.6914 \\
\hline$Q_{\mathrm{FMI}}$ & 0.6459 & 0.4683 & 0.6592 & 0.5376 & 0.6476 & 0.8457 \\
\hline$Q_{\mathrm{SD}}$ & 48.4643 & 56.5707 & 50.2559 & 46.4979 & 55.2796 & 56.1166 \\
\hline$Q_{\mathrm{NCIE}}$ & 0.8060 & 0.8052 & 0.8158 & 0.8059 & 0.8056 & 0.8072 \\
\hline$Q_{\mathrm{MI}}$ & 0.7060 & 0.5567 & 1.2565 & 0.6608 & 0.6660 & 0.8220 \\
\hline$Q_{\mathrm{FMI}}$ & 0.7331 & 0.4911 & 0.7967 & 0.6345 & 0.7229 & 0.8275 \\
\hline$Q_{\mathrm{SD}}$ & 59.4432 & 75.1043 & 59.4872 & 55.5982 & 71.4376 & 89.1413 \\
\hline$Q_{\mathrm{NCIE}}$ & 0.8039 & 0.8030 & 0.8055 & 0.8049 & 0.8035 & 0.8062 \\
\hline$Q_{M I}$ & 0.5698 & 0.3652 & 0.6721 & 0.6271 & 0.5077 & 0.8241 \\
\hline$Q_{\mathrm{FMI}}$ & 0.7380 & 0.5254 & 0.7510 & 0.6657 & 0.7431 & 0.8608 \\
\hline$Q_{\mathrm{SD}}$ & 35.3903 & 46.0797 & 49.6000 & 44.3124 & 45.5952 & 51.4487 \\
\hline
\end{tabular}

4.2. Analysis of Experimental Results. In the example of anatomical and anatomical image fusion, we can see that the anatomical information of the bones or soft tissues is contained in the fused images by the six algorithms; still, differences between fused images can be clearly distinguished, such as focal regions blur (Figures 6(a), 6(c), and $6(d))$, information of soft tissues regions are missing (Figures 6(b) and 6(e)). Our method obtains better performance than other methods. The example of anatomical and functional image fusion shows that the fused images obtained by GFF and CSCS lead to the loss of color information (Figures $7(\mathrm{a}), 7(\mathrm{c})$, and $7(\mathrm{~d})$ ), and the NSCTMSF-PCNN and LP-SR algorithms lead to poor visual effect, for instance, the details of the anatomical image are lost (Figures 7(b) and 7(e)). From the comparisons, our proposed algorithm demonstrates more advantages than the existing algorithms.

Tables 1 and 2 give the objective evaluation results of the proposed MCA-CS-PCNN fusion algorithm and five fusion methods via using objective fusion quality metrics. We mark 
best results employing the boldface in each row. Table 1 shows an objective evaluation of the fused image about anatomical image and functional image. We $Q_{\mathrm{SF}}$ values are only slightly lower than LP-SR in the second pair of images of Figure 4. Our method achieves the significant superiority. From Table 2, it can be see that $Q_{M I}$ values are only slightly lower than GFF in the second pair of images of Figure 5, and the values of $Q_{\mathrm{FMI}}$ and $Q_{\mathrm{SD}}$ in our proposed algorithm demonstrate advantages.

\section{Conclusion}

This paper proposes a perceptual medical image fusion framework based on morphological component analysis combining convolutional sparsity and pulse-coupled neural network, which is called MCA-CS-PCNN for short. It is basically based on the visual system feature that the cat visual cortex can produce complex stimulation, and the neuron representations of complex stimulation can be represented using sparse coding. To this end, we first decomposed source images into cartoon components and texture components by morphological component analysis, and convolutional sparse representation of cartoon layers and texture layers is obtained by prelearned dictionaries. Then, convolutional sparsity is employed to stimulate the PCNN for processing cartoon layers and texture layers. Finally, the medical fused image is computed via combining fused cartoon layers and texture layers. The experimental results verify that the proposed model can produce high performance, which is superior to the state-of-the-art fusion strategy.

\section{Data Availability}

The data used to support the findings of this study can be downloaded from http://www.med.harvard.edu/AANLIB/ home.html.

\section{Conflicts of Interest}

The authors declare that they have no conflicts of interest.

\section{Acknowledgments}

This work was supported by the National Natural Science Foundation of China (no. 82001912), Project funded by the China Postdoctoral Science Foundation (2018M642325), and $\mathrm{Xu}$ Zhou Science and Technology Program, China (KC19146).

\section{References}

[1] R. C. Krempien, S. Daeuber, F. W. Hensley, M. Wannenmacher, and W. Harms, "Image fusion of CT and MRI data enables improved target volume definition in 3Dbrachytherapy treatment planning," Brachytherapy, vol. 2, no. 3, pp. 164-171, 2003.

[2] A. C. Paulino, W. L. Thorstad, and T. Fox, "Role of fusion in radiotherapy treatment planning," Seminars in Nuclear Medicine, vol. 33, no. 3, pp. 238-243, 2003.
[3] R. R. Nair and T. Singh, "Multi-sensor medical image fusion using pyramid-based DWT: a multi-resolution approach," IET Image Processing, vol. 13, no. 9, pp. 1447-1459, 2019.

[4] J. Wang, X. Li, Y. Zhang, and X. Zhang, "Adaptive decomposition method for multi-modal medical image fusion," IET Image Processing, vol. 12, no. 8, pp. 1403-1412, 2018.

[5] Y. Na, L. Zhao, Y. Yang, and M. Ren, "Guided filter-based images fusion algorithm for CT and MRI medical images," IET Image Processing, vol. 12, no. 1, pp. 138-148, 2018.

[6] V. S. Petrovic and C. S. Xydeas, "Gradient-based multiresolution image fusion," IEEE Transactions on Image Processing, vol. 13, no. 2, pp. 228-237, 2004.

[7] G. Qu, D. Zhang, and P. Yan, "Medical image fusion by wavelet transform modulus maxima," Optics Express, vol. 9, no. 4, pp. 184-190, 2001.

[8] L. Yang, B. L. Guo, and W. Ni, "Multimodality medical image fusion based on multiscale geometric analysis of contourlet transform," Neurocomputing, vol. 72, no. 1-3, pp. 203-211, 2008.

[9] Y. Yang, S. Tong, S. Huang, and P. Lin, "Log-Gabor energy based multimodal medical image fusion in NSCT domain," Computational and Mathematical Methods in Medicine, vol. 2014, Article ID 835481, 12 pages, 2014.

[10] G. Bhatnagar, Q. M. J. Wu, and Z. Liu, "A new contrast based multimodal medical image fusion framework," Neurocomputing, vol. 157, pp. 143-152, 2015.

[11] Y. Liu, S. Liu, and Z. Wang, "A general framework for image fusion based on multi-scale transform and sparse representation," Information Fusion, vol. 24, pp. 147-164, 2015.

[12] Z. Wang and Y. Ma, "Medical image fusion using $\mathrm{m}-\mathrm{PCNN}$," Information Fusion, vol. 9, no. 2, pp. 176-185, 2008.

[13] L. Tang, J. Qian, L. Li, J. Hu, and X. Wu, "Multimodal medical image fusion based on discrete tchebichef moments and pulse coupled neural network," International Journal of Imaging Systems and Technology, vol. 27, no. 1, pp. 57-65, 2017.

[14] S. Das and M. K. Kundu, "NSCT-based Multimodal medical image fusion using pulse-coupled neural network and modified spatial frequency," Medical \& Biological Engineering \& Computing, vol. 50, no. 10, pp. 1105-1114, 2012.

[15] P. Ganasala and V. Kumar, "Multimodality medical image fusion based on new features in NSST domain," Biomedical Engineering Letters, vol. 4, no. 4, pp. 414-424, 2014.

[16] M. Yin, X. Liu, Y. Liu, and X. Chen, "Medical image fusion with parameter-adaptive pulse coupled neural network in nonsubsampled shearlet transform domain," IEEE Transactions on Instrumentation and Measurement, vol. 68, no. 1, pp. 49-64, 2019.

[17] C. X. Huang, G. Tian, Y. Lan et al., "A new pulse coupled neural network (PCNN) for brain medical image fusion empowered by shufed frog leaping algorithm," Frontiers Neuroscience, vol. 13, pp. 1-10, 2019.

[18] X. Jin, G. Chen, J. Hou, Q. Jiang, D. Zhou, and S. Yao, "Multimodal sensor medical image fusion based on nonsubsampled shearlet transform and S-PCNNs in HSV space," Signal Processing, vol. 153, pp. 379-395, 2018.

[19] E. T. Rolls and M. J. Tovee, "Sparseness of the neuronal representation of stimuli in the primate temporal visual cortex," Journal of Neurophysiology, vol. 73, no. 2, pp. 713-726, 1995.

[20] D. Ferster, S. Chung, and H. Wheat, "Orientation selectivity of thalamic input to simple cells of cat visual cortex," Nature, vol. 380, no. 6571, pp. 249-252, 1996. 
[21] B. A. Olshausen and D. J. Field, "Emergency of simple-cell receptive field proper ties by learning a sparse code for natural images," Nature, vol. 381, pp. 607-609, 1994.

[22] J.-L. Starck, M. Elad, and D. Donoho, "Redundant multiscale transforms and their application for morphological component separation," Advances in Imaging and Electron Physics, vol. 132, pp. 287-348, 2004.

[23] Y. Jiang and M. Wang, "Image fusion with morphological component analysis," Information Fusion, vol. 18, pp. 107118, 2014.

[24] Y. Liu, X. Chen, R. K. Ward, and Z. Jane Wang, "Image fusion with convolutional sparse representation," IEEE Signal Processing Letters, vol. 23, no. 12, pp. 1882-1886, 2016.

[25] S. Li, X. Kang, and J. Hu, "Image fusion with guided filtering," IEEE Transactions on Image Processing: a Publication of the IEEE Signal Processing Society, vol. 22, no. 7, pp. 2864-2875, 2013.

[26] R. Shen, I. Cheng, and A. Basu, "Cross-scale coefficient selection for volumetric medical image fusion," IEEE Transactions on Bio-Medical Engineering, vol. 60, no. 4, pp. 1069-1079, 2013.

[27] H. Liu and I. Heynderickx, "A perceptually relevant no-reference blockiness metric based on local image characteristics," EURASIP Journal on Advances in Signal Processing, vol. 2009, pp. 1-14, 2009.

[28] H. Liu, N. Klomp, and I. Heynderickx, "A no-reference metric for perceived ringing artifacts in images," IEEE Transactions on Circuits and Systems for Video Technology, vol. 20, no. 4, pp. 529-539, 2010.

[29] S. Wang, K. Gu, X. Zhang, W. Lin, S. Ma, and W. Gao, "Reduced-reference quality assessment of screen content images," IEEE Transactions on Circuits and Systems for Video Technology, vol. 28, no. 1, pp. 1-14, 2018.

[30] K. Gu, J. Qiao, X. Min, G. Yue, W. Lin, and D. Thalmann, "Evaluating quality of screen content images via structural variation analysis," IEEE Transactions on Visualization and Computer Graphics, vol. 24, no. 10, pp. 2689-2701, 2018.

[31] V. Jakhetiya, K. Gu, W. Lin, Q. Li, and S. P. Jaiswal, "A prediction backed model for quality assessment of screen content and 3-D synthesized images," IEEE Transactions on Industrial Informatics, vol. 14, no. 2, pp. 652-660, 2018.

[32] H. Chen and P. K. Varshney, "A human perception inspired quality metric for image fusion based on regional information," Information Fusion, vol. 8, no. 2, pp. 193-207, 2007.

[33] M. B. A. Haghighat, A. Aghagolzadeh, and H. Seyedarabi, "A non-reference image fusion metric based on mutual information of image features," Computers \& Electrical Engineering, vol. 37, no. 5, pp. 744-756, 2011.

[34] S. Li, J. T. Kwok, and Y. Wang, "Combination of images with diverse focuses using the spatial frequency," Information Fusion, vol. 2, no. 3, pp. 169-176, 2001.

[35] Q. Wang, Y. Shen, and J. Jin, "Performance evaluation of image fusion techniques," in Image Fusion: Algorithms and Applications, T. Stathaki, Ed., pp. 469-492, Elsevier, Amsterdam, Netherlands, 2008.

[36] N. Cvejic, C. N. Canagarajah, and D. R. Bull, "Image fusion metric based on mutual information and tsallis entropy," Electronics Letters, vol. 42, no. 11, pp. 626-627, 2006. 